\title{
Editorial
}

\section{Nanomechanics and Nanostructured Multifunctional Materials: Experiments, Theories, and Simulations}

\author{
Jun Lou ${ }^{1}$ and Junlan Wang ${ }^{2}$ \\ ${ }^{1}$ Department of Mechanical Engineering and Materials Science, Rice University, Houston, TX 77005, USA \\ ${ }^{2}$ Department of Mechanical Engineering, University of California - Riverside, Riverside, CA 92521, USA
}

Correspondence should be addressed to Jun Lou, jlou@rice.edu

Received 30 April 2008; Accepted 30 April 2008

Copyright (c) 2008 J. Lou and J. Wang. This is an open access article distributed under the Creative Commons Attribution License, which permits unrestricted use, distribution, and reproduction in any medium, provided the original work is properly cited.

The rapid advances of modern technology require a thorough understanding of mechanics of materials, structures, and processes at the micro- and nanoscales. Carbon nanotubes, nanowires, nanocomposites, nanoscale thin films and coatings, micro- and nano electromechancial systems (MEMS and NEMS), micro- and nanofabrication processes are all examples of materials systems, technologies, and processes whose continuous advancement depends upon fundamental understandings of material properties at small length scales. In order to help them fulfill their promises, mechanics at nanometer length scale must be developed to understand the deformation and failure mechanisms of these small material structures. In addition, advances in the development of new materials for multifunctional applications will require design, fabrication, and characterization at the nanometer length scale. Nanostructured materials that can be tailored to achieve greater mechanical properties along with their electrical, optical, thermal, and other functional properties are essential for future applications in many industry sectors. To achieve the overwhelming potential of nanostructured multifunctional materials, advances in the synthesis of materials with multifunctional capabilities through incorporating nanoscale constituents such as nanotubes, nanofibers, and nanoclays, as well as the development of novel methods and techniques to characterize the mechanical and multifunctional behavior of these materials under application conditions are clearly of critical importance.

The goal of this special issue is to provide a platform for researchers working at the frontier of nanomechanics and nanostructured multifunctional materials to present and discuss exciting new developments on various topics in this area, which include work focused on experiments, theories, and simulations. The special issue with a total of twenty-two papers covers a wide range of topics related to fabrication of nanoscale building blocks such as nanosphere, nanowires, nanorods, and nanoscale thin films; development and application of nanomechanical characterization methods for nanomaterials and other advanced characterization techniques to probe important physical/chemical properties of multifunctional nanomaterial systems; processing and property evaluation of various polymer, ceramic, and metalbased nanocomposites; and finally nanomechanical modeling and simulations from continuum micromechanicsbased modeling to molecule dynamic-based simulations. We sincerely hope such a broad representation of important topics in these vibrant and fast growing research areas would be of interest to the readership.

\section{ACKNOWLEDGMENTS}

The editors would like to acknowledge those who assisted in putting together this special issue, including our invited and contributing authors, reviewers, staff members of Hindawi Publisher, and the Editor in-Chief of Journal of Nanomaterials, Dr. Michael Z. Hu.

Jun Lou

Junlan Wang 

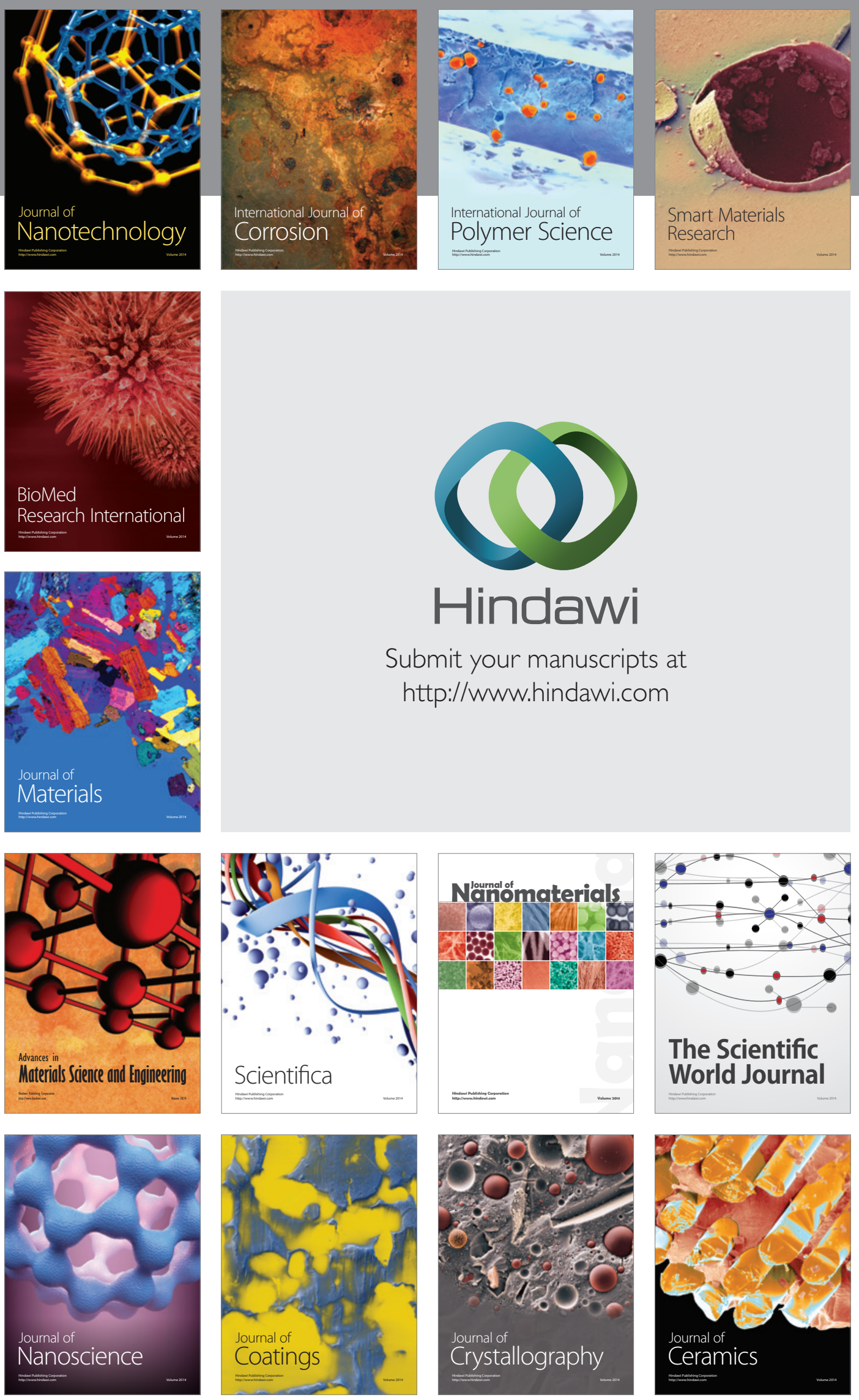

The Scientific World Journal

Submit your manuscripts at

http://www.hindawi.com

\section{World Journal}

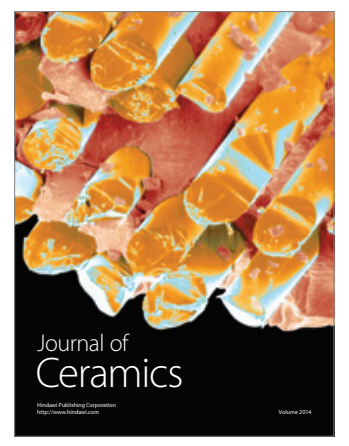

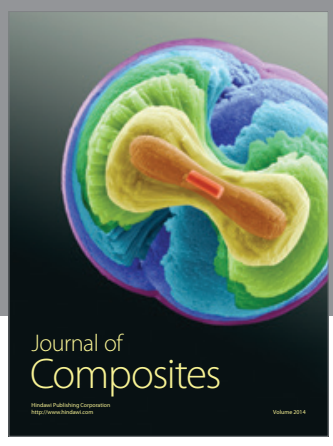
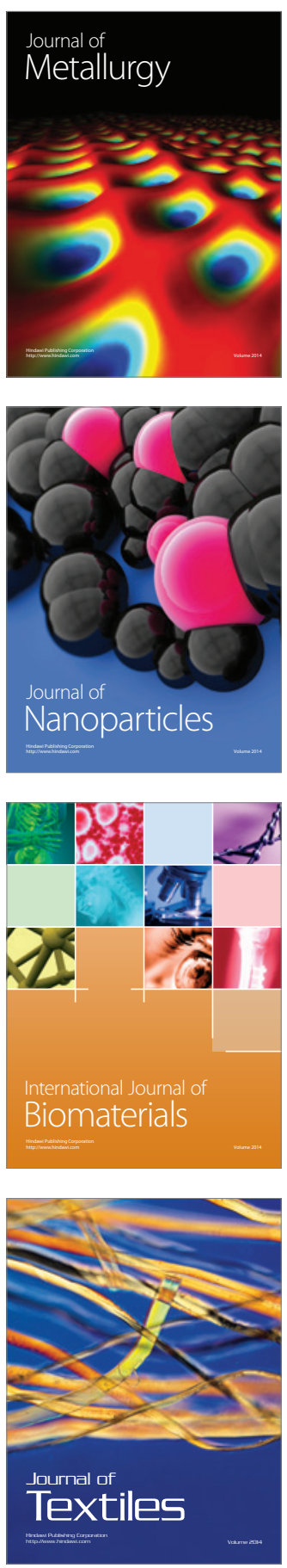